\section{Tiotropium Respimat increases the risk of mortality}

\author{
Christine R Jenkins, ${ }^{1}$ Richard Beasley ${ }^{2}$
}

In the 10 years since tiotropium was introduced into clinical practice, it has become the mainstay long-acting bronchodilator for maintenance treatment in Chronic Obstructive Pulmonary Disease (COPD). Multiple randomised controlled trials have demonstrated its efficacy as an intervention which improves lung function, quality of life, symptom control and exercise capacity, and reduces exacerbations in patients with COPD. ${ }^{1}$

Subsequent to the introduction of tiotropium, evidence emerged from the Lung Health Study that ipratropium, the shortacting anticholinergic medication widely used prior to tiotropium, was associated with a greater risk of cardiac death in the smoking intervention-ipratropium (SI-A) arm compared with the smoking intervention-placebo (SI-P) arm. ${ }^{2}$ There was a preponderance of arrhythmias as a cause of hospitalisation in the SI-A group because of a relatively high prevalence of supraventricular tachycardia. Because smoking cessation rates were similar in both groups, ipratropium looked to be the likely cause.

Following this, the United States Food and Drug Administration, being made aware of an increased risk of stroke in pooled data from 29 trials of tiotropium through a report supplied to it by the manufacturer, issued an early warning in March $2008 .^{3}$ Later that year, a meta-analysis of 17 trials (ipratropium $(n=5)$ and tiotropium $(n=12))$ showed an increased risk of the primary composite endpoint of cardiovascular death, myocardial infarction or stroke (relative risk 1.60, 95\% CI 1.22 to 2.10 ) and of all-cause mortality (relative risk 1.29, 95\% CI 1.00 to 1.65$){ }^{4}$ Inhaled anticholinergics significantly increased the risk of myocardial infarction (relative risk 1.52, 95\% CI 1.04 to 2.22 ) and cardiovascular death (relative risk 1.92, 95\% CI 1.23 to 3.00 ), but not stroke (relative risk 1.46, $95 \%$ CI 0.81 to 2.62 ).

However, also later in 2008 the Understanding Potential Long-Term Impacts

\footnotetext{
${ }^{1}$ Airways Group, Woolcock Institute of Medical Research, Camperdown, Australia; ${ }^{2}$ Medical Research Institute of New Zealand, Wellington, New Zealand

Correspondence to Professor Christine R Jenkins, Department of Thoracic Medicine, Concord Hospital, Hospital Road, Concord, NSW 2139, Australia; christine.jenkins@sydney.edu.au
}

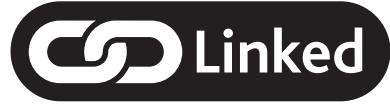

- http://dx.doi.org/10.1136/thoraxjnl-2012-201926 - http://dx.doi.org/10.1136/thoraxjn-2012-202071 - http://dx.doi.org/10.1136/thoraxjn-2012-202483

on Function with Tiotropium (UPLIFT) study, ${ }^{5}$ the largest randomised trial of tiotropium, showed that in comparison with placebo tiotropium was not associated with an increased risk of mortality (HR 0.89, $95 \%$ CI 0.79 to 1.02 ), myocardial infarction (relative risk $0.73,95 \%$ CI 0.53 to 1.00 ) or stroke (relative risk $0.95,95 \%$ CI 0.70 to 1.29 ). Although not adjusted for multiple comparisons, a sensitivity analysis showed a statistically significant reduction in all-cause mortality at the end of the protocol-defined treatment period. These reassuring findings together with the reduction in COPD exacerbations and improvement in lung function and quality of life contributed to a favourable efficacy/safety profile being established for tiotropium. However, the reassurance was tempered by recognition that subjects at the greatest risk of cardiovascular events (moderate to severe renal impairment or a recent history of myocardial infarction, unstable or lifethreatening cardiac arrhythmias, or hospitalisation with heart failure) were excluded from the study, thereby limiting the generalisability of the findings. ${ }^{6}$

Into this uncertainty entered a new device, the Respimat, a fine mist inhaler delivering the tiotropium dose independently of inspiratory effort and patient coordination, and with the potential benefits of a small aerosol particle size and hence more uniform delivery to smaller airways. $^{7}$ In 2011, the British Medical Journal $(B M /)$ published a systematic review and meta-analysis of randomised placebo-controlled trials of tiotropium solution using the mist inhaler (tiotropium Respimat) in the treatment of COPD. ${ }^{8}$ It reported a $50 \%$ increased risk of mortality with tiotropium Respimat. To gain insight into the clinical and public health significance of these findings, it is worthwhile examining the study in more detail, the critiques that have been proposed, the potential mechanisms of the effect and further data that have been published in the 12 months since its publication.
The systematic review and metaanalysis included all parallel group, randomised, placebo-controlled trials of tiotropium Respimat in the treatment of COPD that reported data on mortality and were of at least 30 days duration. ${ }^{8}$ The relative risk of all-cause mortality was estimated using a fixed effect metaanalysis and heterogeneity was assessed with the $\mathrm{I}^{2}$ statistic. In total, five trials were included, two of 12 weeks and three of 12 months duration, with a total of 6522 subjects studied. Tiotropium Respimat significantly increased the risk of all-cause mortality (relative risk 1.52, $95 \%$ CI 1.06 to $\left.2.16, \mathrm{p}=0.02, \mathrm{I}^{2}=0 \%\right)$ and cardiovascular death (relative risk $2.05,95 \%$ CI 1.06 to 3.99). The findings were suggestive of a dose-response effect on all-cause mortality with relative risks of 1.46 (95\% CI 1.01 to 2.10 ) and 2.15 (95\% CI 1.03 to 4.51 ) estimated for the 5 and $10 \mu \mathrm{g}$ preparations, respectively. The overall estimates were not substantially changed by sensitivity analyses using the random effects model, limiting the analysis to three trials of 1 year's duration each, or the inclusion of additional preliminary data on tiotropium Respimat from an unpublished study. In an accompanying editorial it was calculated that one excess death could be expected for every 121 patients with COPD treated with the $5 \mu \mathrm{g}$ dose by Respimat for 12 months. $^{9}$

Six main criticisms have been advanced regarding the meta-analysis and its interpretation. $^{10}{ }^{11}$ First, Boehringer Ingelheim, the manufacturer of tiotropium Respimat, challenged the validity of the analyses; however, two independent systematic reviews and meta-analyses undertaken since the original $B M J$ publication have reported a similar significant increased risk of all-cause mortality with tiotropium Respimat. ${ }^{1} 12$ The Cochrane Database of Systematic Reviews reported a Peto's OR of 1.47 (95\% CI 1.04 to 2.08$)^{1}$ and the recent systematic review and mixed treatment comparison meta-analysis of Dong and colleagues reported an OR of 1.51 (95\% CI 1.06 to 2.19). ${ }^{12}$ Thus, it is reasonable to conclude that the findings of the original $B M J$ publication are robust.

The second critique that the data relating to the $10 \mu \mathrm{g}$ tiotropium Respimat preparation should not be included in the primary analysis because it is not marketed contradicts the Cochrane Handbook which recommends the amalgamation of all relevant treatment arms into one group. ${ }^{13}$ The findings relating to the $10 \mu \mathrm{g}$ tiotropium Respimat preparation also allow investigation of a potential dose-response 
relationship. This is relevant to the clinical situation of moderate to severe renal impairment, which leads to a doubling in plasma tiotropium concentrations. ${ }^{14}$ Thus, some older patients with COPD and moderate to severe renal impairment (with creatine clearance $<50 \mathrm{ml} / \mathrm{min}$ ) who take the $5 \mu \mathrm{g}$ preparation may have a similar or higher systemic exposure to tiotropium as a patient with normal renal function self-administering the tiotropium $10 \mu \mathrm{g}$ preparation.

The third critique that the causes of death were 'diverse,' is weak, as that is the nature of the composite endpoint of 'allcause mortality' and there was a significant increased risk of cardiovascular death. The importance of cardiovascular risk was also suggested by the observation that in the largest randomised controlled trial, COPD patients with known cardiac disease or rhythm disorders were at a particularly greater risk of cardiac death with tiotropium Respimat (relative risks 4.0, $95 \%$ CI 1.2 to 14.1 , and 8.6 , 95\% CI 1.1 to 67.2 , respectively). ${ }^{15}$

The fourth critique concerns the potential effect of a higher withdrawal rate with placebo introducing bias from different periods of treatment exposure, follow-up and selective outcome reporting, an issue also raised by the Cochrane Review. ${ }^{1}$ This potential bias was minimised by the steps taken by the trial investigators to ascertain the vital status of all randomised patients, even if they had discontinued treatment, thereby achieving almost complete capture of the mortality outcomes. ${ }^{8}$

The fifth critique which was also comprehensively addressed in the $B M J$ publication $^{8}$ was that the increase in mortality could be attributed to a baseline imbalance in risk created by randomisation or an unusually low rate of mortality in the placebo arm of these trials. A systematic failure of randomisation is highly unlikely as both the tiotropium Respimat and the placebo groups had comparable baseline characteristics in all the trials, with adequate allocation concealment during randomisation.

The sixth critique is that the findings from the tiotropium Respimat studies may be flawed in some way as the risk was not observed in the larger UPLIFT study in which there was no increased risk of mortality. Certainly, there is a significant difference in risk of mortality between the Handihaler and Respimat inhaler as reported in the two independent meta-analyses. ${ }^{1}{ }^{12}$ There are two main possibilities that may account for this. First, the Respimat device may result in greater systemic exposure to tiotropium. Higher peak plasma concentrations have been demonstrated with the $10 \mu \mathrm{g}$ dose by Respimat compared with the $18 \mu \mathrm{g}$ tiotropium dose from the Handihaler, although the comparative data relating to the $5 \mu \mathrm{g}$ dose via Respimat is less consistent. ${ }^{16} 17$ The other possibility is that the tiotropium Respimat studies used less restrictive exclusion criteria than those employed in UPLIFT, resulting in a greater potential to include patients with renal impairment and cardiovascular disease who are at increased risk for cardiac death with tiotropium. However, this interpretation is limited to some degree by the inconsistent reporting of the inclusion and exclusion criteria in the tiotropium Respimat studies between the study protocols and the published manuscripts. Additionally, overarching exclusion criteria such as 'those with disease who might be at risk because of participation' are very sensitive to investigator and centre effects, resulting in selection bias that may be impossible to ascertain post hoc, and making it very difficult to determine which patients were excluded.

So what are the main studies that have been published since the BMJ publication? In addition to the Cochrane Database Review, ${ }^{1}$ a systematic review and mixed treatment comparison meta-analysis of randomised controlled trials of medications used in the treatment of COPD has been published. ${ }^{12}$ This study reported that tiotropium Respimat increased the risk of death compared with commonly used inhaled medications for COPD including tiotropium Handihaler (OR 1.65, 95\% CI 1.13 to 2.43 ), long-acting $\beta$-agonists (OR 1.63 , 95\% CI 1.10 to 2.44 ) and long acting beta-agonist and inhaled corticosteroid combination therapy (OR 1.90, 95\% CI 1.28 to 2.86). The risk was more evident for cardiovascular death, in patients with severe COPD, and at a higher daily dose.

The other relevant study, presented at the recent European Respiratory Society Meeting in Vienna in September 2012, provided evidence from a Dutch General Practice database that the use of tiotropium Respimat in clinical practice may be causing excess deaths in patients with COPD. ${ }^{18}$ Use of Respimat was associated with an increased risk of dying (HR 1.52, $95 \%$ CI 1.24 to 1.87 ) and this association remained upon adjustment (HR 1.33, 95\% CI 1.07 to 1.65 ).

Thus, the original $B M J$ publication has withstood intensive critical review and with the subsequent publication of the two independent meta-analyses ${ }^{1} 12$ there can be considered to be Level 1 scientific evidence that tiotropium Respimat increases the risk of cardiovascular and all-cause mortality. The accompanying data from the General Practice database suggest that the findings are generalisable to standard clinical practice. ${ }^{18}$ Although the mechanisms of increased cardiovascular events and mortality are not yet fully elucidated, there are plausible explanations which align with the known characteristics of anticholinergic drugs and their effects on cardiac physiology, along with the different deposition and pharmacokinetics of tiotropium delivered by mist inhaler. ${ }^{6}$

This evidence has implications for prescribers, regulatory authorities and the manufacturer. Tiotropium is currently available in the Handihaler device for which the clinical efficacy and safety have been confirmed in COPD patients without concomitant moderate to severe renal impairment, history of myocardial infarction, unstable or life-threatening cardiac arrhythmias, or recent hospitalisation with heart failure. In the absence of evidence of greater clinical benefit with tiotropium Respimat compared with tiotropium Handihaler, ${ }^{19}$ a recommendation can be made that the 5 and $10 \mu \mathrm{g}$ preparations of tiotropium Respimat should not be prescribed in the treatment of COPD.

\section{Funding None.}

Competing interests $\mathrm{RB}$ received funding support from Boehringer Ingelheim to present at a symposium. CJ has recently been invited to join a Boehringer Ingelheim Advisory Board.

Provenance and peer review Commissioned; internally peer reviewed.

To cite Bush A, Pavord ID. Thorax 2013, 68, 6-8.

Accepted 25 October 2012

Thorax 2013;68:6-8.

doi:10.1136/thoraxjnl-2012-202482

\section{REFERENCES}

1 Karner C, Chong J, Poole P. Tiotropium versus placebo for chronic obstructive pulmonary disease. Cochrane Database Syst Rev 2012;7: CD009285.

2 Anthonisen N, Connet JE, Enright PL. Manfreda J and the lung health study research group. Hospitalizations and mortality in the lung health study. Am J Respir Crit Care Med 2002;166: 333-9.

3 Michele TM, Pinheiro S, lyasu S. The safety of tiotropium-the FDA's conclusions. N Engl J Med 2010;363:1097-99.

4 Singh S, Loke YN, Furberg CD. Inhaled anticholinergics and risk of major adverse cardiovascular events in patients with chronic obstructive pulmonary disease. A systematic review and meta-analysis. JAMA 2008;300:1439-50. (Erratum in JAMA 2009:301:1227-30)

5 Tashkin DP, Celli B, Senn S, et al. UPLIFT study investigators. A 4-year trial of tiotropium in chronic obstructive pulmonary disease. $N$ Engl J Med 2008:359:1543-54.

6 Singh S, Loke YK, Enright P, et al. Pro-arrhythmic and pro-ischaemic effects of inhaled anticholinergic medications. Thorax 2012. doi:10.1136/ thoraxjnl-2011-201275 
7 Anderson P. Use of Respimat ${ }^{\circledR}$ Soft MistTM Inhaler in COPD patients. Int I Chron Obstruct Pulmon Dis 2006; 1:251-9.

8 Singh S, Yoon KL, Enright PL, et al. Mortality associated with tiotropium mist inhaler in patients with chronic obstructive pulmonary disease : a systematic review and meta-analysis of randomized controlled trials. BMJ 2011;342:d3215.

9 Cates CJ. Safety of tiotropium: indirect evidence suggests the respimat inhaler is riskier than the handihaler. BMJ 2011;342:d2970.

10 Disse B, Metzdorf N, Martin A, et al. Mortality associated with tiotropium mist inhaler? A critical appraisal of the authors' selection and use of previously communicated tiotropium respimat data. BMJ 2011;342:d3215.

11 Singh S, Loke YL, Enright P, et al. Mortality associated with tiotropium mist inhaler? A critical appraisal of the authors' selection and use of previously communicated tiotropium Respimat data. BMJ 2011:342:d3215.
12 Dong Y-H, Lin H-H, Shau W-Y, et al. Comparative safety of inhaled medications in patients with chronic obstructive pulmonary disease: systematic review and mixed treatment comparison meta-analysis of randomized controlled trials. Thorax 2012. doi: 10.1136/thoraxjnl-2012-201926

13 Higgins JPT, Green S. Cochrane handbook for systematic reviews of interventions. Version 5.1.0. http://www.cochrane-handbook.org/ (accessed Aug 2012).

14 MedSafe, Ministry of Health, Wellington, New Zealand. Tiotropium Handihaler Datasheet. http://www.medsafe.govt.nz/profs/datasheet/s/ Spirivacap.pdf (accessed Aug 2012).

15 Pulmonary-Allergy Drugs Advisory Committee Meeting, US Food and Drug Administration. Clinical Briefing Document, November 192009. NDA \# 21-395 SpirivaHandihaler. http://www.fda. gov/downloads/advisorycommittees/ committeesmeetingmaterials/drugs/ pulmonary-allergydrugsadvisorycommittee/ ucm 190463.pdf (accessed Aug 2012).

16 van Noord JA, Cornelissen PJ, Aumann JL, et al. The efficacy of tiotropium administered via Respimat ${ }^{\circledR}$ Soft Mist inhaler or HandiHaler in COPD patients. Respir Med 2009;103:22-9.

17 Ichinose M, Fujimoto T, Fukuchi Y. Tiotropium $5 \mu \mathrm{g}$ via Respimat and $18 \mu \mathrm{g}$ via HandiHaler; efficacy and safety in Japanese COPD patients. Respir Med 2010:104:228-36.

18 Verhamme K, Afonso A, Romio S, et al. Increased risk of mortality in COPD patients using tiotropium Respimat vs tiotropium Handihaler. European Respiratory Society Annual Scientific Meeting; September 2012. Abstract 196.

19 Ram FSF, Carvallho CR, White J. Clinical effectiveness of the Respimat inhaler device in managing chronic obstructive pulmonary disease: evidence when compared with other handheld inhaler devices. Int I COPD 2011;6: 129-39. 


\section{Corrections}

Jenkins CR, Beasley R. Tiotropium Respimat increases the risk of mortality. Thorax 2013;68:57. doi:10.1136/thoraxjnl-2012-202482. This article's citation information in the pdf should read: To cite Jenkins CR, Beasley R. Thorax 2013;68:5-7. doi:10.1136/ thoraxjnl-2012-202482.

Thorax 2013;68:162. doi:10.1136/thoraxjnl-2012-202482corr1 